\title{
Voice Guided Robot using LabVIEW
}

\author{
Harivardhagini $\mathrm{S}^{1}$, Pranavanand $\mathrm{S}^{2}$ and Ghali Bharadwaja Sharma ${ }^{3}$ \\ ${ }^{1}$ C.V.R College of Engineering, Ibrahimpatan, R.R District, Andhra Pradesh, India \\ Email:harivardhagini@gmail.com \\ ${ }^{2}$ VNR Vignana Jyothi Institute of Engineering \& Technology, Andhra Pradesh, India \\ Email:pranavanands@vnrvjiet.in \\ 3 C.V.R College of Engineering, Ibrahimpatan, R.R District, Andhra Pradesh, India \\ Email:ghali.bharadwaj@gmail.com
}

\begin{abstract}
The main aim of this paper is to develop an efficient and low cost voice guided robot that navigates based on the voice signals given by the human users using LabVIEW 2011 for speech recognition and RF module for wireless transmission. The module is aimed at navigating in environments with noise and other disturbances very effectively. The speech recognition is based on LabVIEW 2011 .NET frameworks and the VI designed is highly flexible and can suit to large variety of applications where in the changes are to be in the microcontroller programming and the hardware requirements which are relevant to the application being designed. The communication to the hardware has been established via RS232 cables using microcontrollers to the transmission kit and the RF signal to communicate with the receiving kit mounted on the mobile robot. Unlike the costly DAQs these hardware communication are highly cost effective and the systems are flexible and can be altered to any utility. Adequate measures are taken to reduce the problems of external disturbances like noise etc. so that the module will understand the command even in very noisy environments. The purpose of these modules is to reduce the human loss due interventions in hazardous environments such as probation of life under debris, bomb detection in target sites etc.
\end{abstract}

Index Terms-Speech recognition, LabVIEW 2011.NET frameworks, RF communication.

\section{INTRODUCTION}

As the control action performed by understanding the voice commands of the humans brings in highly convenient interaction with robots, we have designed multi- utility voice recognition. The implementation of this voice guided robot involves both hardware and software, where in the general block diagram of the complete execution is as follows:
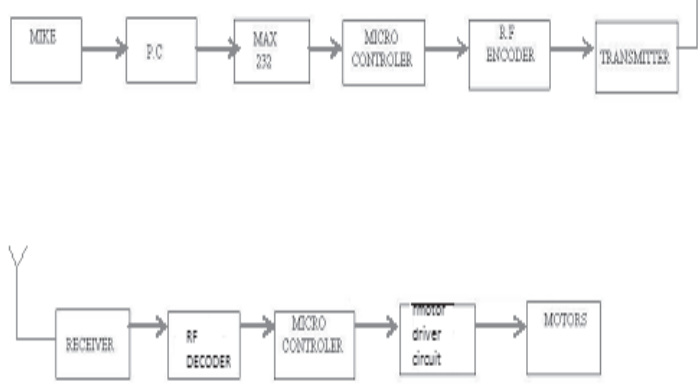

Figure 1.Block diagram

\section{DESCRIPTION}

The microphone will initially receive the voice signal from the human user. The output of this is received by the $P C$ via $3.5 \mathrm{~mm}$ jack. This received information is initiated onto to the LabVIEW using the appropriate constructor and is programmed to perform desired actions. The information from the $\mathrm{PC}$ has to communicate to the $\mathrm{PCB}$ or to the hardware for which a serial communication has to be established. This is done by using a USB to RS232 conversion cable, and the RS232 port is then connected to the MAX232 present on PCB which converts TT Logic to Binary Logic. The information from MAX232 is the received by the micro-controller and will transmit the signal according to program via RF Transmitter. The RF Receiver will receive the information and is given to the micro-controller and the necessary action taking by the controller by initiating the driver IC to drive the motors.

Thus as we give the voice commands the robot will move continuously by following the above algorithm.

\section{IMPLEMENTATION}

In this paper the LabVIEW programming and the micro controller coding are the major software programming involved. The LabVIEW programming involves the speech recognition and the speech to text conversion along with the serial communication.

\section{A.The LabVIEW Programming}

The constructor speech recognition engine is selected as the primary constructor and it is set up to a default audio device. Then it is initialized by the grammar to make the system understand the voice commands by the user.

The commands are predefined here to reduce the noise and other disturbances, the commands here are: Go Forward, Go Backward, Turn Left, Turn Right and Stop.

By the above commands the grammar is built. It is then connected to the text output. This text output is then given to case structure that will finally be displayed as the text box on the front panel of VI.Further this string output is connected to another case structure that will assign the action that is specified in the micro controller. The output of the above case structure is then connected to the serial communication part of the program that will write the information using the VISA serial communication modules present in LabVIEW. This is then given to the transmission kit that is connected to the PC which will finally transmit the encoded signal using the encoder. 

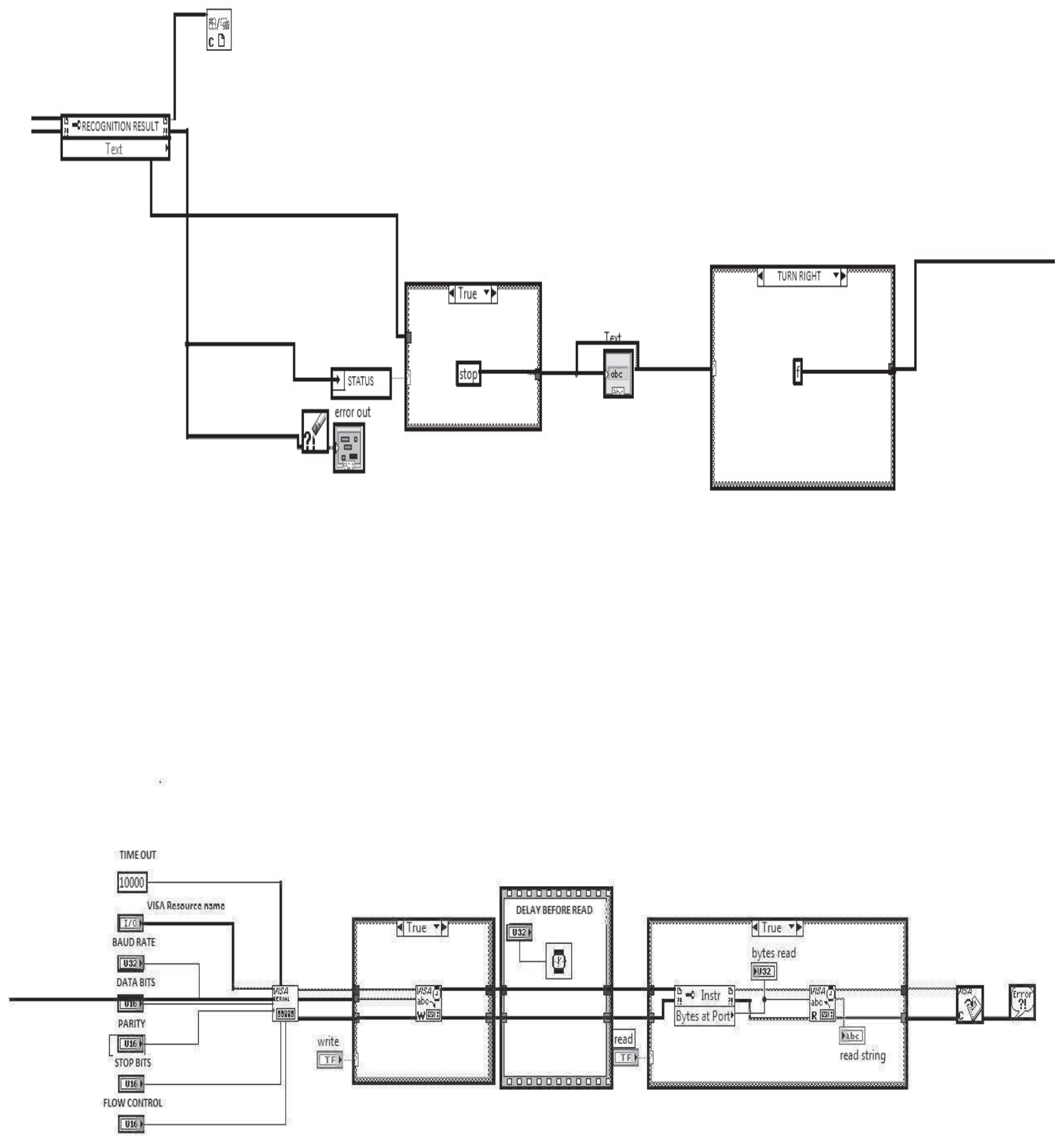

Figure 2. Block Diagram of module 


\section{RF MODULE}

The RF module was built with a transmitter and a receiver. Two micro controllers are used here, one in the transmitter section and the other in the receiving section. In the transmitter the micro controller is coded to receive the information the $\mathrm{PC}$ and transmit the same via RF Transmitter. The second microcontroller is coded to receive the information from the receiver and take the necessary actions using the driver IC to drive the motors.

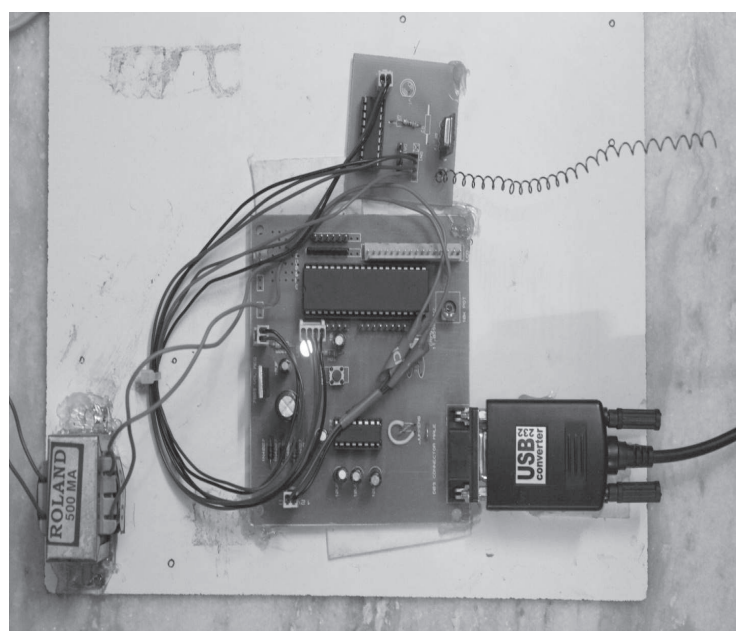

Figure 3. Transmitting side

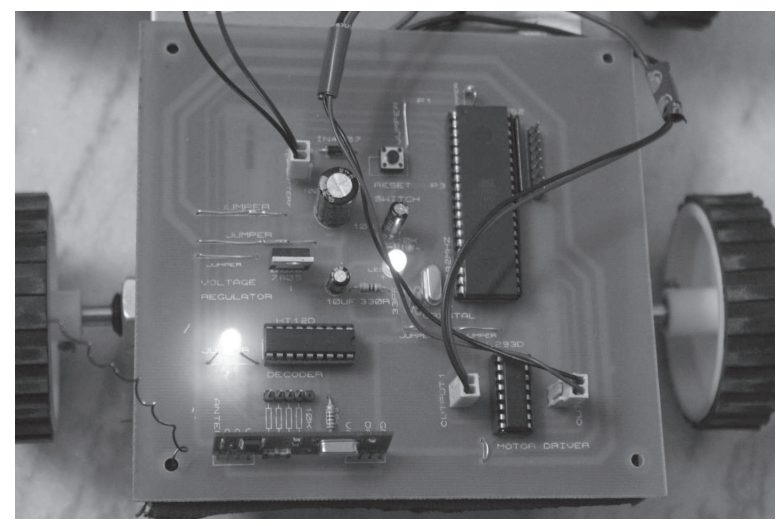

Figure 4 Receiving side

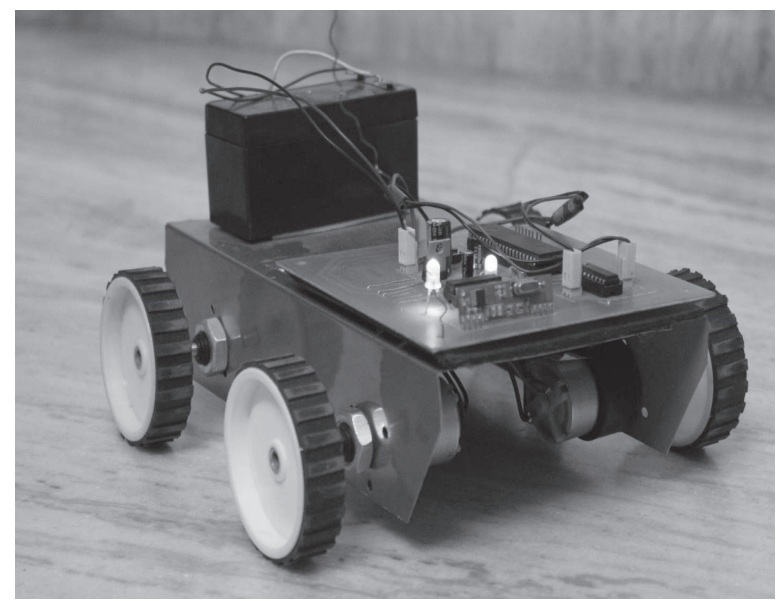

Figure 5. The Robot
This is the final prototype Robot with four metal gear dc motors connected to the wheels and the receiving kit is mounted over it. It has a battery $(12 \mathrm{~V}$, $1.3 \mathrm{Ah}$ ) mounted on the robot which the supply to both robot and the kit.

\section{RESULT AND CONCLUSION}

The codes are deployed onto the micro controller and the serial communication is established. The voice commands are received and the robot navigates on the surface continuously for the commands specified.

The VI and the trans-receiving kit which is designed in this is aimed at lowest cost possible in the vicinage. This requires no specialized DAQs which are available in the market, and are very expensive too. This can be installed at various domestic, industrial, military, medical and many other fields as per the requirement.Though there are various hardware modules that performs this speech recognition, this LabVIEW based module is most advantageous as it is a software and can altered according then there innumerable times. Moreover the hardware will require higher maintenance and is prone to damage at hazardous situations. This module is highly flexible and can be used to perform any task by just making few changes in the commands and the hardware which has to be altered accordingly. Enhancements like addition of cameras, sensors, detectors etc. can be made suit the requirements. The programs can be run in lower platforms also, as well as on Desktops and Laptops. It is flexible on various OS environments like Microsoft windows, Apple Mac, Ubuntu (Linix) etc.

\section{REFERENCES}

[1] Barry E. Paton 1999," LabVIEW Graphical Programming for Instrumentation". Prentice Hall PTP, New Jersey, U.S.A

[2] Curtis D. Johnson 1984," Microprocessor-Based Process Control". Prentice-Hall International, IncEnglewood Cliffs, NJ, U.S.A.

[3] DE-LORENZO, 2002, Electronic Laboratory,"(Basic Board To Study Temperature Regulation)".DL.2155RGT1，D1.2155RGT2， Milano, Italy.

[4] Donald R. Coughanowr, 1991,"Process Systems Analysis and Control". McGraw-Hill, Inc., Singapore

[5] Gary W. Johnson, 1994. "LabVIEW Graphical Programming". McGraw-Hill, Inc., New York, U.S.A.

[6] J. Michael Jacob, 1989," Industrial Control Electronics". Prentice-Hall International, Inc., New Jersey, U.S.A.

[7] National Instruments, 2002, LabVIEW Basics Introduction, Course Manual, U.S.A

[8] National Instruments, 1996, "LabVIEW Graphical Programming for Instrumentation". User Manual. New Jersey, U.S.A. 